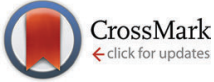

Cite this: Phys. Chem. Chem. Phys., $2015,17,14383$

Received 13th November 2014, Accepted 23rd February 2015

DOI: $10.1039 / c 4 c p 05278 a$

www.rsc.org/pccp

\section{Orbital entanglement and CASSCF analysis of the Ru-NO bond in a Ruthenium nitrosyl complex}

\author{
Leon Freitag, ${ }^{a}$ Stefan Knecht, ${ }^{b}$ Sebastian F. Keller, ${ }^{b}$ Mickaël G. Delcey, ${ }^{c}$ \\ Francesco Aquilante, ${ }^{\mathrm{cd}}$ Thomas Bondo Pedersen, ${ }^{e}$ Roland Lindh, ${ }^{f}$ Markus Reiher ${ }^{\star b}$ \\ and Leticia González*a
}

Complete active space self-consistent field (CASSCF) wavefunctions and an orbital entanglement analysis obtained from a density-matrix renormalisation group (DMRG) calculation are used to understand the electronic structure, and, in particular, the Ru-NO bond of a Ru nitrosyl complex. Based on the configurations and orbital occupation numbers obtained for the CASSCF wavefunction and on the orbital entropy measurements evaluated for the DMRG wavefunction, we unravel electron correlation effects in the Ru coordination sphere of the complex. It is shown that Ru-NO $\pi$ bonds show static and dynamic correlation, while other Ru-ligand bonds feature predominantly dynamic correlation. The presence of static correlation requires the use of multiconfigurational methods to describe the Ru-NO bond. Subsequently, the CASSCF wavefunction is analysed in terms of configuration state functions based on localised orbitals. The analysis of the wavefunctions in the electronic singlet ground state and the first triplet state provides a picture of the Ru-NO moiety beyond the standard representation based on formal oxidation states. A distinct description of the Ru and NO fragments is advocated. The electron configuration of Ru is an equally weighted superposition of $\mathrm{Ru}^{\text {II }}$ and $\mathrm{Ru}^{\mathrm{III}}$ configurations, with the $\mathrm{Ru}^{\mathrm{III}}$ configuration originating from charge donation mostly from $\mathrm{Cl}$ ligands. However, and contrary to what is typically assumed, the electronic configuration of the NO ligand is best described as electroneutral.

\section{Introduction}

The electronic structure and properties of transition metal nitrosyl complexes have been a subject of interest in inorganic and bioinorganic chemistry for a long time. Nitric oxide (NO) plays a role in neurotransmission, blood pressure control and even control of tumour growth. ${ }^{1}$ A number of transition metal nitrosyl complexes have been employed in photodynamical therapy to deliver targeted NO to biological tissues. ${ }^{2}$ Particularly interesting are ruthenium nitrosyls, since they are postulated to be intermediates $^{3,4}$ in the mechanism of action of novel ruthenium anticancer drugs such as NAMI-A ${ }^{5}$ and KP1019. ${ }^{6}$ Understanding the

\footnotetext{
${ }^{a}$ Institut für theoretische Chemie, Universität Wien, Währinger Str. 17, 1090 Vienna, Austria. E-mail: leticia.gonzalez@univie.ac.at

${ }^{b}$ ETH Zürich, Laboratory of Physical Chemistry, Vladimir-Prelog-Weg 2, 8093 Zürich, Switzerland. E-mail: markus.reiher@phys.chem.ethz.ch ${ }^{c}$ Department of Chemistry - Ångström, The Theoretical Chemistry Programme, Uppsala University, Box 518, 75120 Uppsala, Sweden

'Dipartimento di Chimica "G. Ciamician", Università di Bologna, V. F. Selmi 2, 40126 Bologna, Italy

${ }^{e}$ Centre for Theoretical and Computational Chemistry, Department of Chemistry, University of Oslo, P.O. Box 1033 Blindern, 0315 Oslo, Norway

${ }^{f}$ Uppsala Center for Computational Chemistry - UC $C_{3}$, Uppsala University, Box 518, 75120 Uppsala, Sweden
}

electronic structure of the metal-NO moiety is therefore essential to rationalise the mechanisms for NO delivery from nitrosyl complexes and thus obtain a fundamental understanding of the mode of action of ruthenium anti-cancer drugs.

Furthermore, NO is a well-known non-innocent ligand in coordination chemistry, ${ }^{7}$ which leads to an intricate and ambiguous electronic structure of the transition metal nitrosyls. NO can attach to the metal both in a linear or a bent configuration, depending on the electronic structure of the metal, charge of the free NO and the remaining coordination sphere. The metalnitrosyl moiety M-NO usually shows strongly delocalised electron density and a strong covalency. Enemark and Feltham ${ }^{8,9}$ have suggested to describe the electronic structure of this moiety as $\{\mathrm{M}(\mathrm{NO})\}^{n}$, with $n$ being the total number of electrons in the metal $\mathrm{d}$ and nitrosyl $\pi^{*}$ orbitals. Within this framework, however, the electronic character of neither the $\mathrm{Ru}$ nor the NO fragment is known, and it is not possible to assign a particular oxidation state to either fragment. For example, it is unclear whether $\{\mathrm{RuNO}\}^{6}$ structures should be treated as $\mathrm{Ru}^{\mathrm{II}}-\mathrm{NO}^{+}$or $\mathrm{Ru}^{\mathrm{III}}-\mathrm{NO}^{\circ}$. A correct description of the oxidation states of the metal and ligands is important, e.g., for the study of the redox processes involved in the metabolism of redox-active anti-cancer drugs.

Attempts to resolve the ambiguity in the metal-NO bond and to assign oxidation states to the metal and the ligands have 
been carried out in many theoretical, spectroscopic and electrochemical studies. ${ }^{4,10-14}$ It has been largely accepted that most linear $\{\text { RuNO }\}^{6}$ complexes $^{15}$ and many $\{\mathrm{FeNO}\}^{6}$ complexes $^{16}$ are best described as $\mathrm{M}^{\mathrm{II}}-\mathrm{NO}^{+}$. Interestingly, and in contrast to this picture, a recent extensive joint experimental and computational study $^{4}$ on one particular $\{\text { RuNO }\}^{6}$ complex concluded that the physical electronic structure of the $\mathrm{Ru}-\mathrm{NO}$ moiety is better described by $\mathrm{Ru}^{\mathrm{III}}-\mathrm{NO}^{0}$, rather than $\mathrm{Ru}^{\mathrm{II}}-\mathrm{NO}^{+}$.

The majority of computational studies on transition metal complexes employ density functional theory (DFT). ${ }^{17}$ However, many structures belong to the class of the so-called strongly correlated systems which cannot be described by Kohn-Sham DFT $^{18}$ due to its single Slater determinant approximation. In this respect, Kohn-Sham DFT is conceptually similar to HartreeFock theory, which does not incorporate electron correlation (i.e. movement of electrons depending on the instantaneous positions of other electrons). Electron correlation can be classified as ${ }^{19-21}$ dynamic and static (split further into static and nondynamic correlation by some authors ${ }^{19,21}$ ). Dynamic correlation is responsible for keeping the electrons apart and is found in any quantum mechanical system with more than one electron. Static correlation corresponds to significant mixtures of several electronic configurations and is largely present, e.g., in dissociating molecules and many transition metal compounds. While dynamic correlation can be effectively described by DFT and post-Hartree-Fock methods, such as Møller-Plesset perturbation theory or coupled cluster (CC) methods, the proper description of static correlation requires several Slater determinants or configurations in the ansatz. Multiconfigurational methods - such as the complete active space self consistent field (CASSCF), ${ }^{22}$ the restricted active space SCF (RASSCF), ${ }^{23}$ or the density matrix renormalization group $(\mathrm{DMRG})^{24}$ method in its quantum chemical formulation ${ }^{25}$ as well as their corresponding refinements by second-order perturbation theory $^{23,26,27}$ - are then mandatory to describe such systems.

Sizova et al. ${ }^{11}$ were the first to apply a multiconfigurational/ valence-bond treatment to a variety of $\left\{\mathrm{RuNO}^{6}\right.$ complexes. Radon et $a .^{13}$ applied CASSCF localised orbitals and spin densities to analyse the $\mathrm{Fe}-\mathrm{NO}$ bond in several $\{\mathrm{FeNO}\}^{7}$ complexes, as well as calculated the doublet-quartet energy gap with CASPT2/CASSCF. Recently, we have also used CASPT2/CASSCF to study the electronic structure of another $\{\mathrm{RuNO}\}^{6}$ complex. $^{14}$ Boguslawski et al. ${ }^{28}$ compared the CASSCF spin densities of several Fe-NO complexes with DFT results, deeming both unsatisfactory. This unpleasant situation could only be resolved by calculating the spin density from a largeCAS DMRG wavefunction, in which the complete double-d shell correlation effects could be taken into account. ${ }^{29}$ Double-d shell correlation effects are related to the presence of a large number of electrons in compact d shells, resulting in large radial correlation effects in these shells. The second more diffuse $d$ shell gives additional flexibility to describe such correlation effects, and for many $3 \mathrm{~d}$ transition metal compounds the second $\mathrm{d}$ shell must be present to obtain quantitative accuracy with the CASPT2 method. ${ }^{30}$

The last example illustrates the major limitation of CASSCF the factorial growth of computational time with the number of correlating electrons and orbitals. Presently, CASSCF calculations are typically limited to active spaces comprising approximately
16 electrons in 16 orbitals. Over the past few decades, several attempts have been made to overcome the CASSCF factorial scaling problem and allow the usage of larger active spaces: the RASSCF method introduces additional subspaces with a restricted number of excitations; the generalised active space (GAS) ${ }^{31}$ concept takes the RAS concept one step further by introducing an arbitrary number of subspaces. RAS and GAS methods allow us to extend the active spaces at the price of having to choose a restriction of the excitation levels; however this degree of freedom makes these methods less straightforward to use than CASSCF. Recently, the GAS method has been combined with Löwdin's partitioning technique $^{32}$ resulting in the SplitGAS method, ${ }^{33}$ which, despite its demonstrated capability to effectively employ up to $10^{22}$ Slater determinants, still requires algorithmic advances and further development before it can be widely used.

The conceptually different DMRG algorithm employs the reduced density matrix of the system studied to construct and optimise a CAS-like wavefunction, allowing for a polynomial instead of factorial scaling with the number of active orbitals. As a consequence, DMRG allows much larger active spaces than conventional CASSCF, explaining its value for calculations on transition metal complexes dominated by strong static electron correlation $^{34}$ and its remarkable success in transition metal chemistry in recent years. ${ }^{29,35}$

Using the DMRG algorithm, $n$-orbital reduced density matrices are easily obtained from the full density matrix by tracing out contributions from all orbitals in the complementary set of orbitals in the active space. As a consequence, entanglement measures such as the single-orbital entropy ${ }^{36}$ and mutual information ${ }^{37,38}$ calculated from the one-orbital and two-orbital reduced density matrix, respectively, are easily accessible. These orbital-based entanglement measures can be applied to examine the multireference character of the electronic wave function. In particular, they can be correlated with the amount of static and dynamic electron correlation in an electronic wavefunction ${ }^{21}$ or exploited to study chemical bonding in molecule formation and dissociation processes. ${ }^{39}$ Thus, they complement the traditional orbitalbased correlation measures such as the natural orbital and geminal analysis. ${ }^{40}$

In addition to the factorial scaling with the active space size, the cost of multiconfigurational calculations also scales as $\mathrm{O}\left(n^{4}\right)$ with the number of basis functions. Efficient calculations on large molecules, including large transition metal complexes, cannot be performed unless this scaling is reduced. Using approximate representations of the electron repulsion integrals based on density-fitting (DF) and Cholesky decomposition (CD) ${ }^{41}$ reduces the scaling to cubic, thus enabling multiconfigurational calculations on larger molecules. ${ }^{42}$ Analytical gradients employing combined DF and CD techniques extend the applicability to geometry optimisations. ${ }^{43,44}$

In this work we investigate the electronic structure of the $\left\{\mathrm{RuNO}^{6}\right.$ moiety in the trans-[ $\mathrm{RuCl}_{4}(\mathrm{NO})(1 \mathrm{H}$-indazole $\left.)\right]$-complex (RuHIndNO), ${ }^{45}$ which is closely related to the anti-cancer drug KP1019. We perform CASSCF calculations of the $S_{0}$ and $T_{1}$ state of RuHIndNO, describe the electronic structure of the coordination sphere around $\mathrm{Ru}$ and analyse the wavefunction in 
terms of contributions of different configurations and natural orbital occupation numbers. Note that CASPT2/CASSCF singlettriplet energy splittings have been addressed in ref. 44 . Here we also perform orbital entanglement analyses ${ }^{21,38,39}$ based on DMRG calculations of the $S_{0}$ state. We examine different types of electron correlation present in the $\mathrm{Ru}$-ligand bonds and assess the ability of multiconfigurational methods to describe the $\mathrm{Ru}-\mathrm{NO}$ coordination sphere in RuHIndNO.

To shed more light on the electronic structure of the $\{\mathrm{RuNO}\}^{6}$ complex, we transform the CASSCF wavefunctions of the $\mathrm{S}_{0}$ and $\mathrm{T}_{1}$ states into a localised orbital basis and analyse the $\mathrm{Ru}-\mathrm{NO}$ bond and the Ru coordination sphere in terms of configuration state functions (CSFs) based on localised orbitals; we compare the results obtained from the localised orbital analysis to the Mulliken population of $\mathrm{Ru} \mathrm{d}$ orbitals based on both singleconfigurational DFT and the CASSCF wavefunction. At the end of our analysis we adress the non-innocence of the NO ligand: in particular, whether NO is to be considered ionic or neutral and the true $4 \mathrm{~d}$ occupation of the Ru center.

Noting the importance of the double-d shell effects in transition metal compounds, ${ }^{29,30,46}$ we also investigate the double-d-shell effect using entanglement analysis. We perform another DMRG calculation with an active space incorporating another pair of correlating orbitals and a second d shell on $\mathrm{Ru}$.

\section{Computational details}

Geometry optimisation of the lowest singlet $\left(\mathrm{S}_{0}\right)$ and the triplet state $\left(\mathrm{T}_{1}\right)$ has been performed with DFT, using the BP86 functional $^{47,48}$ and the def2-TZVPP basis set. ${ }^{49,50}$ For Ru, the MWB28 effective core potential ${ }^{51}$ (ECP) has been used, and RI-J and MARI-J ${ }^{52}$ approximations were employed for computational efficiency. The triplet geometry has been optimised with the unrestricted Kohn-Sham procedure. The DFT calculations have been performed using the TURBOMOLE $6.5^{53}$ suite of programs.

Using the optimised $S_{0}$ and $T_{1}$ geometries, single-point CASSCF calculations employing the ANO-RCC-VTZP basis set ${ }^{54}$ and atomic compact Cholesky decomposition (acCD)-based algorithms ${ }^{41}$ with the Cholesky decomposition threshold of $10^{-4}$ have been performed with the MOLCAS 7.8 program package. ${ }^{55}$ Mulliken population analyses have been done at the CASSCF and $\mathrm{PBE}^{56}$ levels of theory with the ANO-RCC-VTZP basis set, as implemented in MOLCAS. ${ }^{57}$

The choice of the CASSCF active space is motivated by its feasibility for a configuration analysis of the Ru coordination sphere. Accordingly, all $\mathrm{Ru} 4 \mathrm{~d}$ orbitals and the ligand orbitals interacting with them must be included, resulting in a total active space of 13 orbitals with 16 electrons (denoted $(16,13)$ ), including the five Ru $4 \mathrm{~d}$ orbitals, two pairs of NO $\pi$ and $\pi^{*}$ orbitals, one pair of indazole $\pi$ and $\pi^{*}$ orbitals, one combination of p orbitals on the $\mathrm{Cl}$ atoms (denoted $\sigma_{\mathrm{Cl}}$ ) as well as the NO $\sigma$ orbital. The last two orbitals are particularly important because they participate in the covalent bond formed between the metal and the NO and Cl ligands, respectively; accordingly, each of them mixes with the $d_{z^{2}}$ and the $d_{x^{2}-y^{2}}$ orbitals of the $\mathrm{Ru}$ atom, respectively. A fair comparison of the CASSCF wavefunction analyses on the $S_{0}$ and $T_{1}$ geometry should be done using the same active spaces in both calculations. For RuHIndNO, this can be only achieved in the $\mathrm{S}_{0}$ calculation by a state-average (SA)-CASSCF calculation over the lowest three singlet states. Thus, the $\mathrm{T}_{1}$ calculation was similarly averaged over three states, to ensure that the deterioration of the wavefuction quality due to state averaging is similar in both spin states. The resulting orbitals and corresponding natural orbital occupation numbers of the optimised $\mathrm{S}_{0}$ and $\mathrm{T}_{1}$ geometries are collected in Fig. 1a and b, respectively.

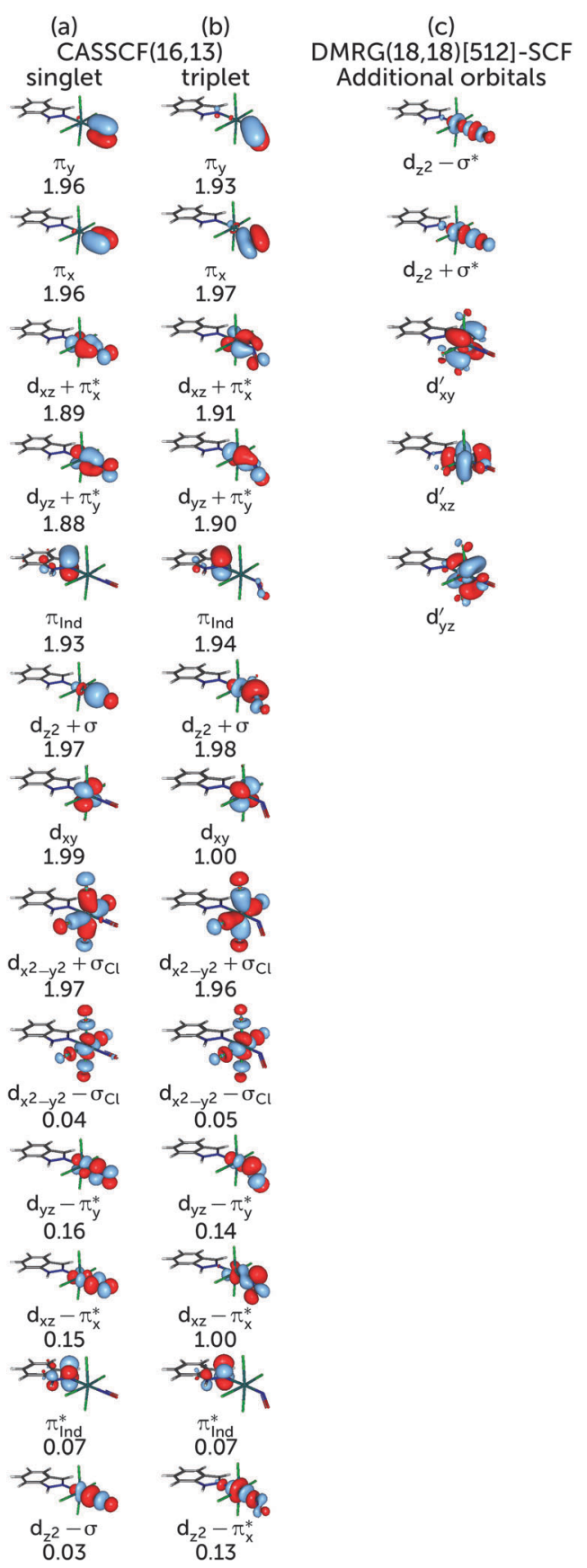

Fig. 1 Active space orbitals and their respective occupation numbers used in the optimization of the $S_{0}(a)$ and $T_{1}$ (b) electronic states using CASSCF calculations. Panel (c) shows the additional orbitals used in the DMRG(18,18)[512]-SCF calculation. Double-shell d orbitals are indicated with a prime. The remaining orbitals correspond to those in column (a). 
For the entanglement measures, a DMRG-CASCI calculation based on the optimised CASSCF orbitals ( $c f$. Fig. 1a) has been performed for the $S_{0}$ state employing the same geometry, active space and basis set as in the CASSCF calculation using the MAQUIS $^{58,59}$ DMRG program, interfaced to the development version of the MOLCAS program package. The number of renormalised active-subsystem states $(m \text {-value })^{58}$ is set to 1000 . With this value, the DMRG calculation reproduces the absolute energy of the CASSCF calculation up to $10^{-6}$ a.u., so that the properties of the DMRG wavefunction can be considered identical to those of the CASSCF wavefunction. This calculation will be denoted DMRG(16,13)[1000], using the shorthand notation $\operatorname{DMRG}\left(n_{\text {electrons }}, n_{\text {orbitals }}\right)[m]$. From the CI-type expansion coefficients of the DMRG wavefunction, a density matrix is constructed, in which environment states can be traced out. These states are states defined on orbitals of the active space that are not considered part of a selected subsystem of orbitals. In the single-orbital case, the selected subsystem consists of only one spatial orbital with four possible states (empty, spin-up, spindown and doubly-occupied) quantum-mechanically embedded into all other orbitals of the active space. States defined on these complementary orbitals are the environment states traced out in the (then) reduced density matrix. The four eigenvalues of this reduced density matrix, $w_{\alpha, i}$, enter a von Neumann entropy expression, which yields the single-orbital entropy, $s(1)_{i}$, for a given orbital $i$, which can be understood as a measure of the interaction of one orbital with all other orbitals:

$$
s(1)_{i}=-\sum_{\alpha} w_{\alpha, i} \ln w_{\alpha, i}
$$

In the same way, a two-orbital entropy, $s(2)_{i, j}$, can be calculated from the sixteen eigenvalues of the reduced density matrix that is valid for the subsystem consisting of the two selected orbitals $i, j$ :

$$
s(2)_{i, j}=-\sum_{\alpha} w_{\alpha, i, j} \ln w_{\alpha, i, j}
$$

As this two-orbital entropy still contains single-orbital-entropy contributions, the two single-orbital entropies may be subtracted, which yields the mutual information, $I_{i, j}$, for orbitals $i$ and $j$ :

$$
I_{i, j}=\frac{1}{2}\left(s(2)_{i, j}-s(1)_{i}-s(1)_{j}\right)\left(1-\delta_{i, j}\right)
$$

The central aspect of these measures is that the quantum entanglement of the states defined on one and two orbitals, respectively, has been properly encoded through the eigenvalues of the reduced density matrices.

To evaluate the correlation contribution of additional orbitals, including the second d shell effect, another DMRG calculation and entanglement analysis was performed with a larger active space. The previous $(16,13)$ active space (Fig. 1a) was augmented with another pair of orbitals consisting of $\mathrm{Ru} \mathrm{d} \mathrm{d}_{z^{2}}$ and $\mathrm{NO} \sigma^{*}$ orbitals and the second $\mathrm{Ru} \mathrm{d}_{x y}, \mathrm{~d}_{x z}$ and $\mathrm{d}_{y z}$ shells ( $c f$. Fig. 1c). From all orbitals not present in the $(16,13)$ active space, these orbitals were expected to give the largest contribution to the correlation in the Ru coordination sphere. The new active space consists of 18 electrons in 18 orbitals. Since this active space is out of reach for the traditional CASSCF implementation,

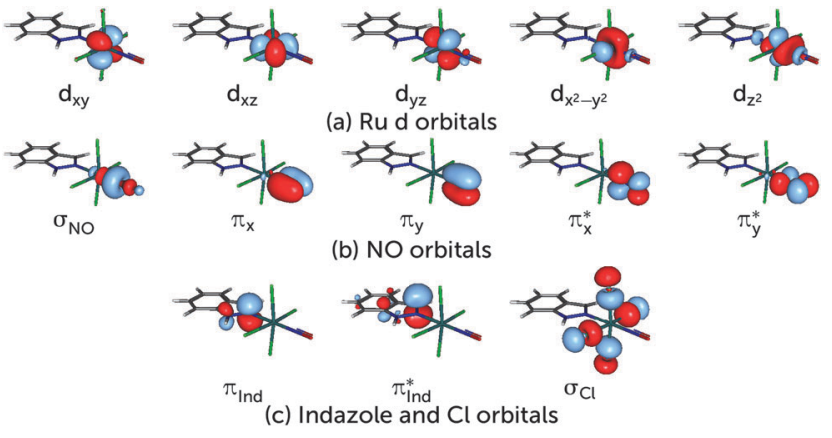

Fig. 2 Localised orbitals for the $S_{0}$ structure.

the orbitals were optimised with the DMRG-SCF approach as implemented in the development version of MOLCAS. We carried out the DMRG $(18,18)[512]-S C F$ orbital optimisation with the smaller ANORCC-MB basis set, augmented with an additional d shell on Ru because the ANO-RCC-VTZP basis set yielded additional p shells on $\mathrm{N}$ or $\mathrm{O}$ atoms of $\mathrm{NO}$ instead of the Ru double shell orbitals. The subsequent DMRG-CASCI step, which is based on the DMRG-SCF orbitals, was done increasing the $m$-value back to 1000 , to be consistent with the DMRG(16,13)[1000] calculation. The orbital entanglement analysis was carried out for the DMRG-CASCI wavefunction, analogous to what has been explained before.

To perform the characterisation of the electronic structure in terms of CSFs based on localised orbitals, all active space orbitals have been localised using the Cholesky algorithm. ${ }^{60}$ As for any rotation among the active orbitals only, this procedure does not change the total energy of the CASSCF wavefunction. The Cholesky localisation yielded orbitals predominantly localised on single atoms, including single p orbitals at the $\mathrm{N}$ and $\mathrm{O}$ atoms of NO. These were converted into a set of proper $\pi$ and $\pi^{*}$ orbitals by forming normalised linear combinations of the form $\frac{1}{\sqrt{2}}\left(P_{\mathrm{N}} \pm P_{\mathrm{O}}\right)$. This procedure yields $\pi$ and $\pi^{*}$ orbitals almost exclusively localised on the NO molecule, and together with the other Cholesky orbitals they form the localised active space. The remaining Cholesky orbitals ( $c f$. Fig. 2) are d orbitals localised on the metal, the $\sigma$ orbital localised at the NO molecule and an orbital consisting of the p orbitals of the four $\mathrm{Cl}$ ligands. This localised active space is used in the discussion of the electronic structure of the complex.

\section{Results and discussion}

Based on the CASSCF wavefunction expressed in natural orbitals (cf. Fig. 1a), the singlet, $\mathrm{S}_{0}$, and triplet, $\mathrm{T}_{1}$, states are predominantly described by the electronic configurations ( $c f$. Fig. 1a)

$$
\begin{aligned}
& \left|\mathrm{S}_{0}\right\rangle=\left|\left(\mathrm{d}_{x z}+\pi_{x}^{*}\right)^{2}\left(\mathrm{~d}_{y z}+\pi_{y}^{*}\right)^{2}\left(\mathrm{~d}_{x y}\right)^{2}\left(\mathrm{~d}_{x z}-\pi_{x}^{*}\right)^{0}\right\rangle \\
& \left|\mathrm{T}_{1}\right\rangle=\left|\left(\mathrm{d}_{x z}+\pi_{x}^{*}\right)^{2}\left(\mathrm{~d}_{y z}+\pi_{y}{ }^{*}\right)^{2}\left(\mathrm{~d}_{x y}\right)^{1}\left(\mathrm{~d}_{x z}-\pi_{x}^{*}\right)^{1}\right\rangle
\end{aligned}
$$

respectively (other active orbitals are, respectively, doubly- or unnocupied). Fig. 3 shows a schematic representation of these dominant configurations. In the $\mathrm{S}_{0}$ linear structure, the $\mathrm{d}_{x z}$ and 
(a)

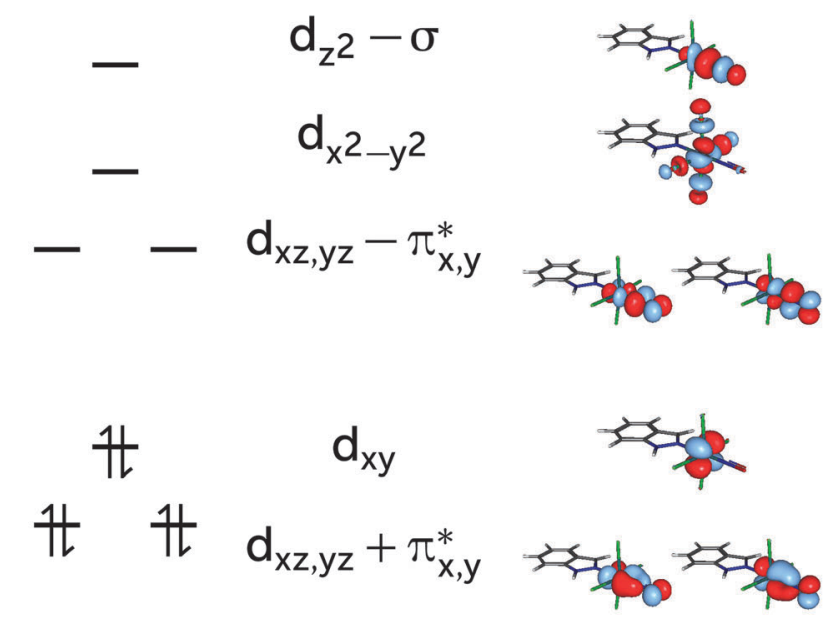

(b)

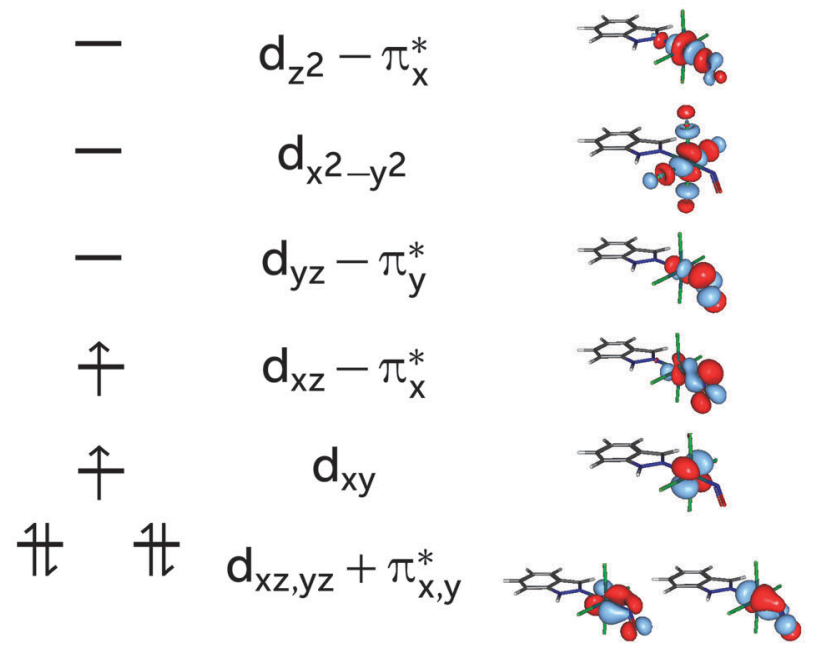

Fig. 3 Principal configurations expressed in terms of CASSCF natural orbitals for the $S_{0}(a)$ and the $T_{1}$ (b) state.

$\mathrm{d}_{y z}$ orbitals of $\mathrm{Ru}$ interact with $\pi^{*}$ orbitals of NO, forming two bonding and two antibonding orbitals, which are denoted $\mathrm{d}_{x z, y z} \pm \pi_{x, y^{*}} . \mathrm{d}_{z^{2}}$ of Ru with the $\sigma$ orbital of NO forms another pair of bonding and antibonding orbitals denoted $\mathrm{d}_{z^{2}} \pm \sigma$ (cf. Fig. 1), again indicating a strongly covalent interaction of $\mathrm{Ru}$ with NO. The triplet dominant configuration is a $\mathrm{d}_{x y} \rightarrow \mathrm{d}_{x z}-\pi_{x}{ }^{*}$ excitation with respect to $\left|S_{0}\right\rangle$. Since the latter orbital is an antibonding orbital, the Ru-NO bond is weaker in the $\mathrm{T}_{1}$ structure than in the $S_{0}$ structure, where the $d_{x y}$ orbital is doubly occupied. Indeed, the bond in the triplet geometry $(1.838 \AA)$ is longer than that of the singlet $(1.718 \AA) .{ }^{44}$ Unlike the linear $\mathrm{S}_{0}$ structure, the interaction of the $d_{z^{2}}$ orbital with the $\pi^{*}$ orbitals of the NO ligand is not symmetry forbidden; therefore a linear combination $\mathrm{d}_{z^{2}}-\pi_{x}{ }^{*}$ is formed.

The major configurations discussed above correspond to $77 \%$ of the singlet and $78 \%$ of the triplet wavefunctions of RuHIndNO. These weights are lower than the typical value of over $90 \%$ for a molecule where the ground state is well described with a single configuration. The remaining $\approx 20 \%$ are distributed among many other configurations, each with weights below $3 \%$. One might then be tempted to conclude that a single-configurational description is sufficient in this case, arguing that the remaining wavefunction contributions are negligible or that they arise due to the dynamic correlation of the system, present in every molecule. If that was the case, however, double excitations would dominate the remaining configurations and single excitations would have much less weight due to the Brilloin theorem. Indeed, one can find that the configuration with the second largest weight of (3\% and $2 \%$ ) in the $\mathrm{S}_{0}$ and $\mathrm{T}_{1}$ wavefunctions, respectively, is a local $\pi \rightarrow \pi^{*}$ double excitation on the indazole ligand; this configuration can be attributed to dynamic correlation between these orbitals. However, a number of single excitations with comparable weight are also present in the wavefunction, for instance, the $\left(\mathrm{d}_{x z}+\pi_{x}^{*}\right) \rightarrow\left(\mathrm{d}_{x z}-\pi_{x^{*}}\right)$ and $\left(\mathrm{d}_{y z}+\pi_{y}{ }^{*}\right) \rightarrow\left(\mathrm{d}_{y z}-\pi_{y}{ }^{*}\right)$ excitations in the $\mathrm{S}_{0}$ wavefunction, and excitations to the $\left(\mathrm{d}_{x z, y z}-\pi_{x, y^{*}}\right)$ and $\mathrm{d}_{z^{2}}-\pi_{x}{ }^{*}$ orbitals, in the $\mathrm{T}_{1}$ state, which points to the presence of static correlation. For comparison, the former contributions amount to $18 \%$ of the $\mathrm{S}_{0}$ wavefunction in the related $\left[\mathrm{Ru}\left(\mathrm{PaPy}_{3}\right)(\mathrm{NO})\right]^{2+}$ complex. ${ }^{14}$

The presence of both static and dynamic correlation in the $\mathrm{Ru}-\mathrm{NO}$ bond of RuHIndNO is also reflected by the occupation numbers of the orbitals involved in the $\mathrm{Ru}-\mathrm{NO}$ bonds, which differ significantly from the formal values of 2 (doubly occupied) and 0 (unoccupied). In the $\mathrm{S}_{0}$ state these are the $\left(\mathrm{d}_{x z, y z}+\pi_{x, y^{*}}\right)$ and $\left(\mathrm{d}_{x z, y z}-\pi_{x, y}{ }^{*}\right)$ orbitals with occupation numbers of $1.89,1.88$, 0.15 and 0.16 respectively ( $c f$. Fig. 1 ). Similar occupation numbers for these orbitals are also found in the $\mathrm{T}_{1}$ state, although here the role of the $\left(\mathrm{d}_{y z}-\pi_{y}{ }^{*}\right)$ orbital is taken over by the $\left(\mathrm{d}_{z^{2}}-\pi_{y^{*}}\right)$ orbital: the occupation number of the former orbital is exactly 1 , which indicates that it does not contribute to the electron correlation. The discrepancies from the formal uncorrelated values of 2 and 0 are also larger than those of the orbitals providing dynamic correlation only, e.g. the $\pi, \pi^{*}$ pair of indazole ( $\pi_{\text {Ind }}$ and $\left.\pi_{\text {Ind }}{ }^{*}\right)(1.93 / 1.94$ and 0.07$)$. Not surprisingly, similar behaviour has been found in $\{\mathrm{FeNO}\}^{7}$ complexes before, ${ }^{13}$ although the effects are even more pronounced there - with occupation numbers of antibonding orbitals as large as 0.3 .

Fig. 4 shows the single-orbital entropies and mutual information for the $S_{0}$ structure, as defined in eqn (1)-(3), as obtained from the DMRG $(16,13)[1000]$ calculation. One can immediately recognise that orbitals $4,5,9$ and 10 (corresponding to the $\mathrm{d}_{x z, y z} \pm \pi_{x, y}{ }^{*}$ orbitals) have the largest single-orbital entropy (as indicated by the size of the corresponding red circles in Fig. 4), while e.g. orbital $3\left(\mathrm{~d}_{x y}\right)$ shows very low entropy. Orbitals 4,5 , 9 and 10 also show high entanglement with each other, and additionally 9 and 10 are also entangled with the $\pi_{x, y}$ orbitals, labelled 1 and 2. Large single-orbital entropies and strong entanglement with more than one orbital are a signature of static correlation. In contrast, small single-orbital entropies combined with weak entanglement among many orbitals or strong entanglement between two orbitals only is an indication of dynamic correlation. Accordingly, the $\pi_{x, y}-\mathrm{d}_{x z, y z}-\pi_{x, y}{ }^{*}$ orbitals $(1,2,4,5,9,10)$, corresponding to two $\mathrm{Ru}-\mathrm{NO} \pi$ bonds, are strongly entangled 


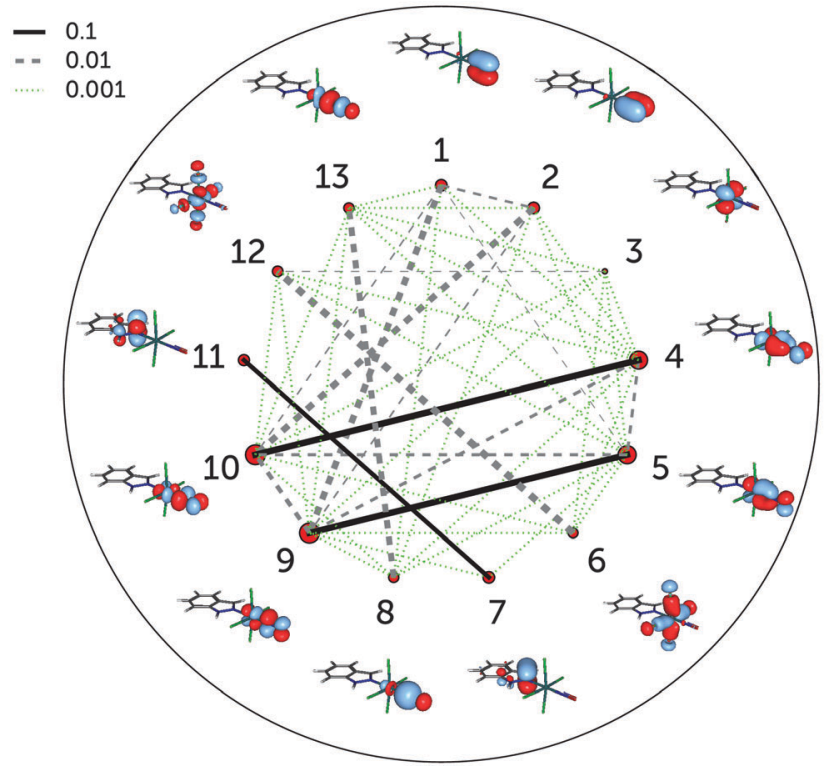

Fig. 4 Single-orbital entropy, $s(1)$, and mutual information, $l$, in the DMRG(16,13)[1000] (equivalent to the CASSCF) wavefunction of RuHIndNO. The size of the red circles next to the orbitals correlates with the magnitude of the corresponding single-orbital entropy. The lines connecting the dots represent the mutual information: solid lines indicate strong entanglement $(I>0.1)$, dashed grey lines stand for middle entanglement $(0.01>I>0.1)$ and dotted green lines indicate weak entanglement $(0.001>I>0.01)$. The line width is also proportional to the absolute value of $I$.

(i.e. interact strongly) and are responsible for static correlation. The entanglement of the orbitals 1 with 9 and 2 with 10 is due to dynamic correlation, as expected from $\pi \pi^{*}$ pairs. One can distinguish other orbital pairs which show largely dynamic correlation, i.e. have smaller single-orbital entropy and are strongly entangled only with each other, but not with other orbitals of the active space, such as orbitals 7 and $11\left(\pi_{\text {Ind }}\right.$ and $\pi_{\text {Ind }}{ }^{*}$, which are again a textbook example of dynamic correlation), 6 and $12\left(\mathrm{~d}_{x^{2}-y^{2}} \pm \sigma_{\mathrm{Cl}}\right)$ and 8 and $13\left(\mathrm{~d}_{z^{2}} \pm \sigma\right)$. The latter two orbital pairs correspond to $\mathrm{Ru}$ bonds with chlorido ligands and the $\mathrm{Ru}-\mathrm{NO} \sigma$ bond. The single-orbital entropy values correlate well with the deviation of the occupation numbers from 2 or 0 (recall Fig. 1a). The orbitals with the largest deviation $(4,5$, $9,10)$ show both static and dynamic correlation, whereas orbitals with smaller deviations ( 7 and 11, 6 and 12, 8 and 13) show mostly dynamic correlation.

The incorporation of the additional $\mathrm{d}_{z^{2}}-\mathrm{NO} \sigma^{*}$ pair and the double-shell d orbitals in the active space (DMRG $(18,18)[1000]$ calculation) does not change the entanglement picture (Fig. 5). Compared to DMRG(16,13)[1000], only a few weak interactions with the newly added orbitals can be seen. The $\mathrm{d}_{z^{2}}-\mathrm{NO} \sigma$ pair (orbitals 8 and 13) has similar single-orbital entropies to the newly added $\mathrm{d}_{z^{2}}$-NO $\sigma^{*}$ orbitals (14 and 15$)$ and is weaklyentangled with them; similarly weak is the interaction of the $\mathrm{d}_{x y}$ orbital (3) with its double shell (16). The entanglement of the two other double shells is even smaller - they are not affecting the entanglement in the Ru-NO bond in any way. Single-orbital entropies of other orbitals, present in the smaller active space, remain also unaffected. The few additional weak interactions

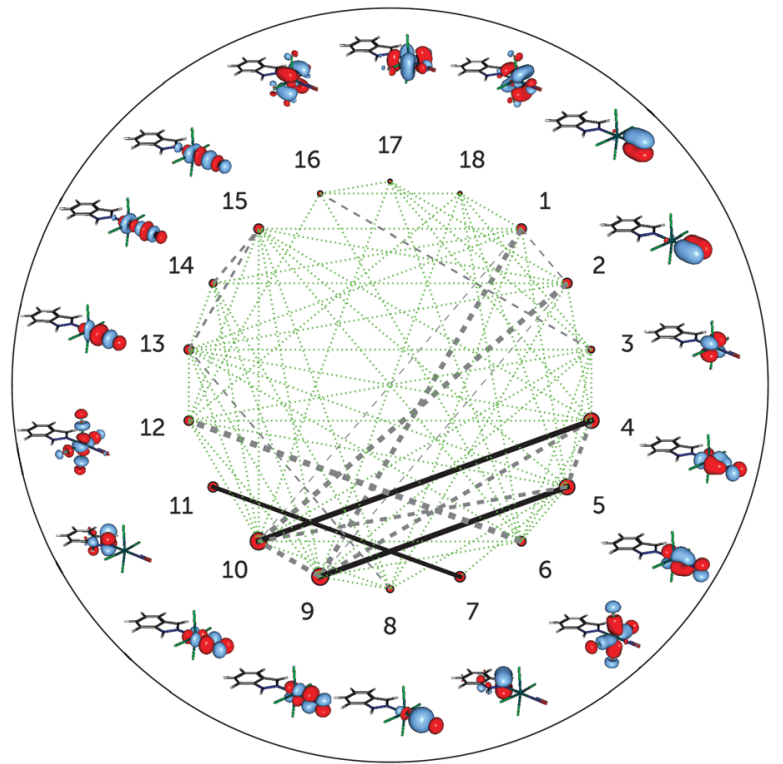

Fig. 5 Single-orbital entropy, $s(1)$, and mutual information, $l$, for the $\operatorname{DMRG}(18,18)[1000]$ wavefunction. Labels as in Fig. 4; additional orbitals have been labelled $14-18$

added with the extension of the active space thus should be attributed to the dynamic correlation and do not affect the overall entanglement picture of the $\mathrm{Ru}$ coordination sphere found with the smaller $(16,13)$ active space. The lack of strong entanglement and small single-orbital entropies of the doubleshell d orbitals shows that their overall effect on the correlation is negligible, similar to what has been found by Pierloot and coworkers for the description of electronic excitations in other $4 \mathrm{~d}$ transition metals. ${ }^{30,46}$ The negligible effect of the doubleshell d orbitals also explains why the orbital optimisation of the DMRG(18,18)[512]-SCF calculation could only be done with the small ANO-RCC-MB basis set, which excludes the additional $\mathrm{p}$ shells of the N, C and O atoms. If the larger ANO-RCC-VTZP basis set is used, the double-shell d orbitals will then be replaced by these p shells. Notably, a similar problem was faced in an earlier study of a ruthenocene complex reported by Phung et al. ${ }^{61}$ We thus emphasise that despite the difference in the basis set, the entanglement picture is similar in both cases.

Summarising, the configurational analysis in terms of CASSCF natural orbitals and the entanglement analyses reveal that the $\mathrm{Ru}-\mathrm{NO} \sigma$ bond and other Ru-ligand bonds exhibit mostly dynamic correlation and therefore they are well described with singleconfigurational methods, whereas the Ru-NO $\pi$ bonds show static correlation and therefore require a multiconfigurational treatment. It is precisely the electronic structure of these $\pi$ bonds that contributes to the non-innocence of the NO ligand and, hence, a multiconfigurational analysis is best suited to determine the electronic structure of the $\{\mathrm{RuNO}\}^{6}$ moiety.

$\mathrm{An}$ attempt to determine the electronic configuration of $\mathrm{Ru}$ can be performed with the help of Mulliken population analysis. For illustrative purposes and for the sake of comparison with the DFT work of Bučinský et al., ${ }^{4}$ we contrast Mulliken population differences of $\mathrm{Ru} 4 \mathrm{~d}$ orbitals from wavefunctions obtained from 
CASSCF and DFT calculations. The total $\mathrm{Ru} 4 \mathrm{~d}$ atomic orbital population of the single-determinantal wavefunction is 6.47 , in very good agreement with the total $\mathrm{Ru} d$ population of 6.54 obtained in a similar Ru nitrosyl complex by Bučinský et al. ${ }^{4}$ The value obtained from the CASSCF wavefunction is 6.02 . Given that a single-determinant wavefunction corresponds to a $\mathrm{Ru}^{\mathrm{II}}\left(\mathrm{d}^{6}\right)-\mathrm{NO}^{+}$configuration, i.e. to a theoretical $\mathrm{Ru} 4 \mathrm{~d}$ occupation of 6 , the Mulliken population analysis "overestimates" the $\mathrm{d}$ population by approximately half an electron (0.47). Assuming that the intrinsic overestimation of the Mulliken analysis is the same for both wavefunctions, a corrected Mulliken $4 \mathrm{~d}$ population for the CASSCF wavefunction can be estimated to be 6.02 $0.47=5.55$ electrons. A d population value below 6 within a multiconfigurational calculation is due to the mixture of $\mathrm{Ru}^{\mathrm{II}}\left(\mathrm{d}^{6}\right)-$ $\mathrm{NO}^{+}$and $\mathrm{Ru}^{\mathrm{III}}\left(\mathrm{d}^{5}\right)-\mathrm{NO}^{0}$ configurations in the wavefunction. Although these values should be considered purely qualitative, the difference in Mulliken populations between single-determinant and the CASSCF wavefunction hints to the need of a multiconfiguration treatment.

Further insight into the $\mathrm{Ru}$ coordination sphere can be gained from transforming the CSFs into the basis of localised orbitals (Fig. 2) and analysing the CASSCF wavefunctions in terms of the transformed CSFs. The $\mathrm{S}_{0}$ wavefunction expressed in terms of localised orbitals results in a very diffuse expansion, with a large amount of configurations having small but comparable weights, none above $6 \%$. The configurations with the highest weights of $6 \%$ and $4 \%$ are $\left(\sigma_{\mathrm{Cl}}\right)^{2}\left(\mathrm{~d}_{x y}\right)^{2}\left(\mathrm{~d}_{y z}\right)^{2}\left(\mathrm{~d}_{x z}\right)^{1}\left(\mathrm{~d}_{z^{2}}\right)^{0}\left(\mathrm{~d}_{x^{2}-y^{2}}\right)^{0}$ $\left(\pi_{\text {Ind }}\right)^{1}\left(\pi_{\text {Ind }}\right)^{1}\left(\sigma_{\mathrm{NO}}\right)^{2}\left(\pi_{x}\right)^{2}\left(\pi_{y}\right)^{2}\left(\pi_{x}^{*}\right)^{1}\left(\pi_{y}^{*}\right)^{0}$ and

$$
\begin{gathered}
\left(\sigma_{\mathrm{Cl}}\right)^{2}\left(\mathrm{~d}_{x y}\right)^{2}\left(\mathrm{~d}_{y z}\right)^{1}\left(\mathrm{~d}_{x z}\right)^{2}\left(\mathrm{~d}_{z^{2}}\right)^{0}\left(\mathrm{~d}_{x^{2}-y^{2}}\right)^{0} \\
\left(\pi_{\text {Ind }}\right)^{1}\left(\pi_{\text {Ind }}\right)^{1}\left(\sigma_{\mathrm{NO}}\right)^{2}\left(\pi_{x}\right)^{2}\left(\pi_{y}\right)^{2}\left(\pi_{x}^{*}\right)^{0}\left(\pi_{y^{*}}\right)^{1}
\end{gathered}
$$

and include the $\mathrm{d}_{x z} \rightarrow \pi_{x}^{*}$ and $\mathrm{d}_{y z} \rightarrow \pi_{y}^{*}$ excitation, respectively, reflecting two $\mathrm{d} \rightarrow \pi_{\mathrm{NO}}{ }^{*}$ back dative bonds along both the $x$ and $y$ axes. Both of these configurations feature five electrons in Ru d orbitals and five electrons in the NO orbitals, which corresponds to a $\mathrm{Ru}^{\mathrm{III}}\left(\mathrm{d}^{5}\right)-\mathrm{NO}^{0}$ character. The configuration with the nextlargest contribution $(3 \%)$ is of a $\mathrm{Ru}^{\mathrm{II}}\left(\mathrm{d}^{6}\right)-\mathrm{NO}^{+}$character:

$$
\begin{gathered}
\left(\sigma_{\mathrm{Cl}}\right)^{2}\left(\mathrm{~d}_{x y}\right)^{2}\left(\mathrm{~d}_{y z}\right)^{2}\left(\mathrm{~d}_{x z}\right)^{2}\left(\mathrm{~d}_{z^{2}}\right)^{0}\left(\mathrm{~d}_{x^{2}-y^{2}}\right)^{0} \\
\left(\pi_{\text {Ind }}\right)^{1}\left(\pi_{\text {Ind }}\right)^{1}\left(\sigma_{\mathrm{NO}}\right)^{2}\left(\pi_{x}\right)^{2}\left(\pi_{y}\right)^{2}\left(\pi_{x}^{*}\right)^{0}\left(\pi_{y}\right)^{0}
\end{gathered}
$$

Due to the large number of contributing configurations, a detailed analysis of the character and contributions to the total wavefunction of each particular configuration is not feasible. Instead, we resort to calculating the collective weights of the configurations corresponding to the particular resonance structure. But rather than calculating only weights of e.g. $\mathrm{Ru}^{\mathrm{II}}-\mathrm{NO}^{+}$to $\mathrm{Ru}^{\mathrm{III}}-\mathrm{NO}^{0}$ configurations (as done in the previous work of Radon et $a .^{13}$ ), we classify the CSFs into several classes based on the occupancy of either Ru or ligands, or the collective occupation of $\mathrm{Ru}$ and some ligands. The relative weights of configurations belonging to each class are shown in Table 1: in the first class, we consider only the occupation of Ru d orbitals ( $c f$. Fig. 2a and Table 1a), then only the NO orbital occupancy (Fig. $2 b$ and Table $1 \mathrm{~b})$ equal to $n=6\left(\mathrm{NO}^{-}\right), 5\left(\mathrm{NO}^{0}\right)$ or $4\left(\mathrm{NO}^{+}\right)$, and finally the combined Ru d and NO occupancy (Table 1c).
Table 1 Contributions of all configurations in the $S_{0}$ and $T_{1}$ states with certain properties: (a) only Ru electron configuration taken into account; (b) Only NO electron configuration taken into account; (c) both Ru and NO electron configurations are taken into account; (d) the charge transfer from $\mathrm{Cl}$ to $\mathrm{NO}$ and $\mathrm{Ru}$ is considered

\begin{tabular}{llcc}
\hline & Character & Contr. $\mathrm{S}_{0}(\%)$ & Contr. $\mathrm{T}_{1}(\%)$ \\
\hline (a) & $\mathrm{Ru}^{\mathrm{IV}}\left(\mathrm{d}^{4}\right)$ & 7.7 & 8.9 \\
& $\mathrm{Ru}^{\mathrm{III}}\left(\mathrm{d}^{5}\right)$ & 42.0 & 44.9 \\
& $\mathrm{Ru}^{\mathrm{II}}\left(\mathrm{d}^{6}\right)$ & 39.9 & 38.4 \\
(b) & $\mathrm{NO}^{-}$ & 18.3 & \\
& $\mathrm{NO}^{0}$ & 58.0 & 23.1 \\
& $\mathrm{NO}^{+}$ & 21.5 & 62.3 \\
& & & 12.2 \\
(c) & $\mathrm{Ru}^{\mathrm{IV}}\left(\mathrm{d}^{4}\right)$ and $\mathrm{NO}^{-}$ & 7.3 & \\
& $\mathrm{Ru}^{\mathrm{III}}\left(\mathrm{d}^{5}\right)$ and $\mathrm{NO}^{0}$ & 31.9 & 8.2 \\
& $\mathrm{Ru}^{\mathrm{II}}\left(\mathrm{d}^{6}\right)$ and $\mathrm{NO}^{+}$ & 14.3 & 8.2 \\
& $\mathrm{Ru}^{\mathrm{II}}\left(\mathrm{d}^{6}\right)$ and $\mathrm{NO}^{0}$ & 24.7 & 28.7 \\
& & & \\
& & 24.6 & 28.7 \\
(d) & $\mathrm{Ru}^{\mathrm{II}}\left(\mathrm{d}^{6}\right)$ and $\left(\sigma_{\mathrm{Cl}}\right)^{1}$ & 10.0 &
\end{tabular}

From the analysis of Table 1 a we see that the contribution of $\mathrm{Ru}^{\mathrm{II}}\left(\mathrm{d}^{6}\right)$ and $\mathrm{Ru}^{\mathrm{III}}\left(\mathrm{d}^{5}\right)$ configurations to the $\mathrm{S}_{0}$ state is almost the same, yielding a formal oxidation state of $\mathrm{Ru}$ of 2.5. This value is in accordance with the corrected Mulliken d population in the CASSCF wavefunction of 5.55 determined previously, despite the fact that Mulliken populations should be treated only qualitatively. A similar process is carried out with NO (Table 1b). The $\mathrm{Ru}^{\mathrm{II}}$ to $\mathrm{Ru}^{\mathrm{III}}$ ratios do not correspond to the ratios of ionic to neutral NO: the $\mathrm{NO}^{0}$ contribution is the predominant one in this complex (58\%). Moreover, $\mathrm{NO}^{+}$contributions are largely cancelled out by $\mathrm{NO}^{-}$contributions.

Since the net charge $(-1)$ of the complex cannot be explained with a $\mathrm{Ru}$ formal oxidation state of 2.5 and a $\mathrm{NO}^{0}$ ligand, we also consider the class of configurations combining the $\mathrm{Ru}$ and $\mathrm{NO}$ occupancies. Table $1 \mathrm{c}$ shows that the $\mathrm{Ru}^{\mathrm{III}}-\mathrm{NO}^{0}$ configurations have the largest collective weight, above $30 \%$, which is 2.2 times as large as that of the $\mathrm{Ru}^{\mathrm{II}}-\mathrm{NO}^{+}$configurations. This weight ratio is slightly smaller than the weight ratio of $\mathrm{NO}^{0}$ to $\mathrm{NO}^{+}$configurations, which is approximately 2.7 . This shows that the $\mathrm{Ru}-\mathrm{NO}$ bond situation is dominated by a strong $\mathrm{d} \rightarrow \pi_{\mathrm{NO}}{ }^{*}$ back donation leading to $\mathrm{NO}^{0}$ and the $\mathrm{d}^{6}$ character of Ru comes from elsewhere. Indeed, we find a large amount of configurations with $\mathrm{Ru}^{\mathrm{II}}\left(\mathrm{d}^{6}\right)$ and $\mathrm{NO}^{0}$ character, with an even larger weight than that of the $\mathrm{Ru}^{\mathrm{II}}\left(\mathrm{d}^{6}\right)$ and $\mathrm{NO}^{+}$configurations. Table $1 \mathrm{~d}$ reveals that these configurations entirely correspond to the $\sigma_{\mathrm{Cl}} \rightarrow \mathrm{d}_{x^{2}-y^{2}}$ excitations, i.e. to a charge transfer from $\mathrm{Cl}$ ligands.

Summarising the configuration analysis for the $S_{0}$ state, we may conclude that the electronic structure of $\left\{\mathrm{RuNO}^{6}\right.$ is a mixture of several important contributions from which the $\mathrm{Ru}^{\mathrm{III}}-\mathrm{NO}^{0}$ configurations are most important, indicating a strong $\mathrm{d} \rightarrow \pi_{\mathrm{NO}}{ }^{*}$ back donation. The $\mathrm{Ru}^{\mathrm{II}}-\mathrm{NO}^{+}$configurations are about half as important if compared by total contributions to the wavefunction, and give $\mathrm{Ru}$ some of its $\mathrm{Ru}^{\mathrm{II}}$ character. Despite the lesser significance of $\mathrm{Ru}^{\mathrm{II}}-\mathrm{NO}^{+}$configurations, $\mathrm{Ru}$ shows a large amount of $\mathrm{Ru}^{\mathrm{II}}$ character, almost equal to its $\mathrm{Ru}^{\mathrm{III}}$ character by having a formal oxidation state of 2.5. A larger amount of the $\mathrm{d}^{6}$ character of $\mathrm{Ru}$, however, does not arise from these configurations, 
but rather from an electron transfer from the $\mathrm{Cl}$ ligands, which can be seen from the contribution of $\mathrm{Ru}^{\mathrm{II}}\left(\mathrm{d}^{6}\right)-\left(\sigma_{\mathrm{Cl}}\right)^{1}$ configurations: this contribution is almost identical to that of $\mathrm{Ru}^{\mathrm{II}}\left(\mathrm{d}^{6}\right)-\mathrm{NO}^{0}$.

Very similar results are obtained for the $\mathrm{T}_{1}$ state, despite its different molecular structure and electronic wavefunction. Most notably, the weights of $\mathrm{Ru} \mathrm{d}^{5}$ and $\mathrm{d}^{6}$ configurations are alike and hence the formal oxidation state of $\mathrm{Ru}$ is also approximately 2.5. The CSFs with the largest weight have the same electronic configurations as in the case of $\mathrm{S}_{0}$, albeit with a different spin and weights under $3 \%$. At first glance, this is quite unexpected since the $T_{1}$ state involves an excitation to a metal-NO $\pi^{*}$ antibonding orbital and $\mathrm{Ru}-\mathrm{NO}$ back donation gets stronger. As such one would expect a withdrawal of electron density from the metal to NO. Indeed, we observe it to some extent, as the weight of $\mathrm{NO}^{+}$and $\mathrm{Ru}^{\mathrm{II}}\left(\mathrm{d}^{6}\right)-\mathrm{NO}^{+}$configurations decreases compared to the $\mathrm{S}_{0}$ state $\left(8.8 \%\right.$ in $\mathrm{T}_{1}$ vs. $14.3 \%$ in $\left.\mathrm{S}_{0}\right)$ : the bent-coordinated NO has even less $\mathrm{NO}^{+}$contributions than the linear-coordinated one. However, this electron withdrawal from the metal is compensated by the stronger $\mathrm{Ru} \rightarrow \mathrm{Cl}$ dative bond: the cumulative weight of $\mathrm{Ru}^{\mathrm{II}}\left(\mathrm{d}^{6}\right)-\left(\sigma_{\mathrm{Cl}}\right)^{1}$ configurations increases to $28.7 \%$. This stronger dative bond compensates for the electron density loss on Ru due to a stronger back donation, yielding a similar $\mathrm{Ru}$ electronic configuration to that in $\mathrm{S}_{0}$.

\section{Conclusion}

In this work, we have employed multiconfigurational methods to analyse the electronic structure of the lowest singlet and triplet states of RuHIndNO, a ruthenium nitrosyl complex. We performed a CASSCF calculation on the optimised geometries for the $S_{0}$ and $T_{1}$ states of RuHIndNO and analysed the resulting wavefunction both in terms of CASSCF natural orbitals and localised orbitals. The Ru-NO bond shows strong electronic correlation, both static and dynamic, which is supported by the weight of the dominant configuration being significantly below 100\%, comparably large weights of single excitations and large fractional populations of the orbitals involved in this bond and the analysis of orbital entanglement. An orbital entanglement analysis based on the oneand two-orbital reduced density matrices calculated from the DMRG wavefunction of the $\mathrm{S}_{0}$ state provides further evidence of strong static correlation in the $\mathrm{Ru}-\mathrm{NO} \pi$ bonds, while the $\mathrm{Ru}-\mathrm{NO} \sigma$ bond and other $\mathrm{Ru}$-ligand bonds are largely dominated by dynamic correlation. An additional entanglement analysis based on a larger active-space calculation shows a negligible effect of the double-shell d orbitals on the static correlation effects. Furthermore, Mulliken $\mathrm{Ru}$ d orbital population based on the single-reference DFT and CASSCF wavefunction shows a discrepancy of approximately 0.5 electrons. In view of these results, we advocate the usage of multiconfigurational methods such as CASSCF to describe the correct bonding situation in the Ru-NO moiety.

CASSCF-type methods also allow for an extensive electronic structure analysis of the metal centre, ligands and metal-ligand bonds in the $\mathrm{Ru}$ coordination sphere. By a comparatively straightforward unitary transformation of the active-space orbitals, an operation which does not change the physical content of the wavefunction, we obtain a possibility to quantify the contributions from different electronic configurations and therefore to describe the electronic structure of $\{\mathrm{RuNO}\}^{6}$ more precisely than any assigned formal oxidation state would do. As we have shown, a single structure e.g. $\mathrm{Ru}^{\mathrm{III}}-\mathrm{NO}^{0}$ or $\mathrm{Ru}^{\mathrm{II}}-\mathrm{NO}^{+}$does not account for the complexity of the $\left\{\mathrm{RuNO}^{6}\right.$ electronic structure. Although the electronic structure of the RuHIndNO complex is a superposition of configurations like $\mathrm{Ru}^{\mathrm{III}}-\mathrm{NO}^{0}, \mathrm{Ru}^{\mathrm{II}}-\mathrm{NO}^{+}$and many others, we gain more details about the structure when we describe the $\mathrm{Ru}$ and NO fragments of the Ru-NO bond separately. In this view, our results show that the electronic configuration of $\mathrm{Ru}$ consists of approximately equal amounts of $\mathrm{d}^{5}\left(\mathrm{Ru}^{\mathrm{III}}\right)$ and $\mathrm{d}^{6}\left(\mathrm{Ru}^{\mathrm{II}}\right)$ contributions, resulting in a formal $\mathrm{Ru}$ oxidation state of 2.5. The NO electronic configuration, on the other hand, shows a predominantly neutral character, which is in contrast to the commonly accepted picture of the $\mathrm{Ru}^{\mathrm{II}}-\mathrm{NO}^{+}$ description. The NO neutral character arises mainly due to the $\mathrm{d} \rightarrow \pi_{\mathrm{NO}}{ }^{*}$ back donation, but a dative contribution by the $\mathrm{Cl}$ ligands compensates the outflux of the electron density due to this back donation. This description of Ru and NO is almost the same for both the $S_{0}$ and $T_{1}$ state, despite the different electronic structures, with the only difference that metal $\rightarrow$ NO back donation is even stronger in the $\mathrm{T}_{1}$ structure. This increase of the metal $\rightarrow$ NO back donation is additionally supported by the increase of the bond length in the triplet state.

\section{Acknowledgements}

Financial support from the University of Vienna, the COST Action CM1305 "Explicit Control Over Spin-states in Technology and Biochemistry”, the Research Council of Norway through a Centre of Excellence Grant (Grant No. 179568/V30), the Italian Ministry of Education and Research (MIUR)-Grant No. RBFR1248UI, the Uppsala University, eSSENCE, and the Swedish Research Council (VR) is gratefully acknowledged. Furthermore, we thank Yingjin Ma for the help with the MAQUIS-MOLCAS interface, as well as Vladimir Arion and Gabriel Büchel for fruitful discussions.

\section{References}

1 (a) E. Culotta and D. E. Koshland, Science, 1992, 258, 1862-1865; (b) F. C. Fang, Nitric Oxide and Infection, Springer, 1999; (c) L. J. Ignarro, Nitric Oxide: Biology and Pathobiology, Academic Press, 2000.

2 (a) P. Ford, J. Bourassa, K. Miranda, B. Lee, I. Lorkovic, S. Boggs, S. Kudo and L. Laverman, Coord. Chem. Rev., 1998, 171, 185-202; (b) P. C. Ford and I. M. Lorkovic, Chem. Rev., 2002, 102, 993-1018; (c) A. R. Butler and I. L. Megson, Chem. Rev., 2002, 102, 1155-1166; (d) J. L. Bourassa and P. C. Ford, Coord. Chem. Rev., 2000, 200-202, 887-900; (e) M. Hoshino, L. Laverman and P. C. Ford, Coord. Chem. Rev., 1999, 187, 75-102; $(f)$ C. F. Works, C. J. Jocher, G. D. Bart, X. Bu and P. C. Ford, Inorg. Chem., 2002, 41, 3728-3739; ( $g$ ) C. F. Works and P. C. Ford, J. Am. Chem. Soc., 2000, 122, 7592-7593; (h) A. K. Patra and P. K. Mascharak, Inorg. Chem., 2003, 42, 
7363-7365; (i) E. Tfouni, D. R. Truzzi, A. Tavares, A. J. Gomes, L. E. Figueiredo and D. W. Franco, Nitric Oxide, 2012, 26, 38-53.

3 B. Serli, E. Zangrando, E. Iengo, G. Mestroni, L. Yellowlees and E. Alessio, Inorg. Chem., 2002, 41, 4033-4043.

4 L. Bučinský, G. E. Büchel, R. Ponec, P. Rapta, M. Breza, J. Kožíšek, M. Gall, S. Biskupič, M. Fronc, K. Schiessl, O. Cuzan, D. Prodius, C. Turta, S. Shova, D. A. Zając and V. B. Arion, Eur. J. Inorg. Chem., 2013, 2505-2519.

5 (a) J. M. Rademaker-Lakhai, D. v. d. Bongard, D. Pluim, J. H. Beijnen and J. H. M. Schellens, Clin. Cancer Res., 2004, 10, 3717-3727; (b) E. Alessio, G. Mestroni, A. Bergamo and G. Sava, Curr. Top. Med. Chem., 2004, 4, 1525-1535.

6 C. G. Hartinger, S. Zorbas-Seifried, M. A. Jakupec, B. Kynast, H. Zorbas and B. K. Keppler, J. Inorg. Biochem., 2006, 100, 891-904.

7 (a) C. Jørgensen, Coord. Chem. Rev., 1966, 1, 164-178; (b) W. Kaim, Eur. J. Inorg. Chem., 2012, 343-348.

8 J. Enemark and R. Feltham, Coord. Chem. Rev., 1974, 13, 339-406.

9 J. H. Enemark and R. D. Feltham, J. Am. Chem. Soc., 1974, 96, 5002-5004.

10 (a) M. Li, D. Bonnet, E. Bill, F. Neese, T. Weyhermüller, N. Blum, D. Sellmann and K. Wieghardt, Inorg. Chem., 2002, 41, 3444-3456; (b) R. G. Serres, C. A. Grapperhaus, E. Bothe, E. Bill, T. Weyhermüller, F. Neese and K. Wieghardt, J. Am. Chem. Soc., 2004, 126, 5138-5153; (c) P. Singh, B. Sarkar, M. Sieger, M. Niemeyer, J. Fiedler, S. Záliš and W. Kaim, Inorg. Chem., 2006, 45, 4602-4609; (d) G. F. Caramori and G. Frenking, Organometallics, 2007, 26, 5815-5825; (e) G. F. Caramori and G. Frenking, Croat. Chem. Acta, 2009, 82, 219-232; $(f)$ G. F. Caramori, A. G. Kunitz, K. F. Andriani, F. G. Doro, G. Frenking and E. Tfouni, Dalton Trans., 2012, 41, 7327-7339; $(g)$ A. G. De Candia, J. P. Marcolongo, R. Etchenique and L. D. Slep, Inorg. Chem., 2010, 49, 6925-6930; (h) J. Conradie and A. Ghosh, J. Phys. Chem. B, 2007, 111, 12621-12624; (i) H.-Y. Cheng, S. Chang and P.-Y. Tsai, J. Phys. Chem. A, 2004, 108, 358-361; $(j)$ J. Oláh and J. N. Harvey, J. Phys. Chem. A, 2009, 113, 7338-7345; (k) K. F. Andriani, G. F. Caramori, F. G. Doro and R. L. T. Parreira, Dalton Trans., 2014, 43, 8792-8804.

11 O. V. Sizova, V. I. Baranovski, N. V. Ivanova and V. V. Sizov, Mol. Phys., 2003, 101, 715-720.

12 M. Radoń and K. Pierloot, J. Phys. Chem. A, 2008, 112, 11824-11832.

13 M. Radoń, E. Broclawik and K. Pierloot, J. Phys. Chem. B, 2010, 114, 1518-1528.

14 L. Freitag and L. González, Inorg. Chem., 2014, 53, 6415-6426.

15 M. J. Rose and P. K. Mascharak, Coord. Chem. Rev., 2008, 252, 2093-2114.

16 F. Roncaroli, M. Videla, L. D. Slep and J. A. Olabe, Coord. Chem. Rev., 2007, 251, 1903-1930.

17 C. J. Cramer and D. G. Truhlar, Phys. Chem. Chem. Phys., 2009, 11, 10757-10816.

18 A. J. Cohen, P. Mori-Sánchez and W. Yang, Science, 2008, 321, 792-794.
19 R. J. Bartlett and J. F. Stanton, in Reviews in Computational Chemistry, ed. K. B. Lipkowitz and D. B. Boyd, John Wiley \& Sons, Inc., 1994, pp. 65-169.

20 O. Sinanoğlu and D. F. Tuan, J. Chem. Phys., 1963, 38, 1740-1748.

21 K. Boguslawski, P. Tecmer, Ö. Legeza and M. Reiher, J. Phys. Chem. Lett., 2012, 3, 3129-3135.

22 B. O. Roos, P. R. Taylor and P. E. Siegbahn, Chem. Phys., 1980, 48, 157-173.

23 P. A. Malmqvist, K. Pierloot, A. R. M. Shahi, C. J. Cramer and L. Gagliardi, J. Chem. Phys., 2008, 128, 204109.

24 (a) S. R. White, Phys. Rev. Lett., 1992, 69, 2863-2866; (b) S. R. White, Phys. Rev. B: Condens. Matter Mater. Phys., 1993, 48, 10345-10356.

25 (a) K. H. Marti and M. Reiher, Z. Phys. Chem., 2010, 224, 583-599; (b) G. K.-L. Chan and S. Sharma, Annu. Rev. Phys. Chem., 2011, 62, 465-481; (c) Y. Kurashige, Mol. Phys., 2013, 112, 1485-1494; (d) S. Wouters and D. van Neck, Eur. Phys. J. D, 2014, 68, 272; (e) T. Yanai, Y. Kurashige, W. Mizukami, J. Chalupský, T. N. Lan and M. Saitow, Int. J. Quantum Chem., 2015, 115, 283-299; $(f)$ S. Sharma and G. K.-L. Chan, J. Chem. Phys., 2012, 136, 124121; (g) Ö. Legeza, R. M. Noack, J. Sólyom and L. Tincani, in Computational Many-Particle Physics, ed. H. Fehske, R. Schneider and A. Weiße, Springer Berlin Heidelberg, 2008, pp. 653-664.

26 K. Andersson, P. A. Malmqvist, B. O. Roos, A. J. Sadlej and K. Wolinski, J. Phys. Chem., 1990, 94, 5483-5488.

27 (a) Y. Kurashige and T. Yanai, J. Chem. Phys., 2011, 135, 094104; (b) S. Sharma and G. K.-L. Chan, J. Chem. Phys., 2014, 141, 111101.

28 K. Boguslawski, C. R. Jacob and M. Reiher, J. Chem. Theory Comput., 2011, 7, 2740-2752.

29 K. Boguslawski, K. H. Marti, Ö. Legeza and M. Reiher, J. Chem. Theory Comput., 2012, 8, 1970-1982.

30 K. Pierloot, in Computational Organometallic Chemistry, ed. T. R. Cundari, Marcel Dekker Inc., New York, 2001, pp. 123-158.

31 D. Ma, G. Li Manni and L. Gagliardi, J. Chem. Phys., 2011, 135, 044128.

32 P.-O. Löwdin, J. Chem. Phys., 1951, 19, 1396-1401.

33 G. Li Manni, D. Ma, F. Aquilante, J. Olsen and L. Gagliardi, J. Chem. Theory Comput., 2013, 9, 3375-3384.

34 K. H. Marti, I. Malkin Ondík, G. Moritz and M. Reiher, J. Chem. Phys., 2008, 128, 014104.

35 (a) Y. Kurashige, G. K.-L. Chan and T. Yanai, Nat. Chem., 2013, 5, 660-666; (b) S. Wouters, T. Bogaerts, P. V. D. Voort, V. V. Speybroeck and D. V. Neck, J. Chem. Phys., 2014, 140, 241103; (c) S. Sharma, K. Sivalingam, F. Neese and G. K.-L. Chan, Nat. Chem., 2014, 6, 927-933; (d) J. Chalupský, T. A. Rokob, Y. Kurashige, T. Yanai, E. I. Solomon, L. Rulíšek and M. Srnec, J. Am. Chem. Soc., 2014, 136, 15977-15991.

36 Ö. Legeza and J. Sólyom, Phys. Rev. B: Condens. Matter Mater. Phys., 2003, 68, 195116.

37 Ö. Legeza and J. Sólyom, Phys. Rev. Lett., 2006, 96, 116401.

38 J. Rissler, R. M. Noack and S. R. White, Chem. Phys., 2006, 323, 519-531. 
39 (a) K. Boguslawski, P. Tecmer, G. Barcza, Ö. Legeza and M. Reiher, J. Chem. Theory Comput., 2013, 9, 2959-2973; (b) M. Mottet, P. Tecmer, K. Boguslawski, Ö. Legeza and M. Reiher, Phys. Chem. Chem. Phys., 2014, 16, 8872-8880.

40 (a) P. Pulay and T. P. Hamilton, J. Chem. Phys., 1988, 88, 4926-4933; (b) M. S. Gordon, M. W. Schmidt, G. M. Chaban, K. R. Glaesemann, W. J. Stevens and C. Gonzalez, J. Chem. Phys., 1999, 110, 4199-4207.

41 (a) N. H. F. Beebe and J. Linderberg, Int. J. Quantum Chem., 1977, 12, 683-705; (b) H. Koch, A. Sánchez de Merás and T. B. Pedersen, J. Chem. Phys., 2003, 118, 9481-9484; (c) T. B. Pedersen, F. Aquilante and R. Lindh, Theor. Chem. Acc., 2009, 124, 1-10; (d) F. Aquilante, L. Boman, J. Boström, H. Koch, R. Lindh, A. Sánchez de Merás and T. B. Pedersen, in Challenges and Advances in Computational Chemistry and Physics, ed. M. G. Papadopoulos, R. Zalesny, P. G. Mezey and J. Leszczynski, Springer, 2011, vol. 13, pp. 301-344.

42 (a) F. Aquilante, T. B. Pedersen, R. Lindh, B. O. Roos, A. Sánchez de Merás and H. Koch, J. Chem. Phys., 2008, 129, 024113; (b) F. Aquilante, P.-Å. Malmqvist, T. B. Pedersen, A. Ghosh and B. O. Roos, J. Chem. Theory Comput., 2008, 4, 694-702; (c) F. Aquilante, T. K. Todorova, T. B. Pedersen, L. Gagliardi and B. O. Roos, J. Chem. Phys., 2009, 131, 34113; (d) D. Roca-Sanjuán, F. Aquilante and R. Lindh, WIREs Comput. Mol. Sci., 2012, 2, 585-603.

43 (a) F. Aquilante, R. Lindh and T. B. Pedersen, J. Chem. Phys., 2008, 129, 034106; (b) J. Boström, F. Aquilante, T. B. Pedersen and R. Lindh, J. Chem. Theory Comput., 2013, 9, 204-212.

44 M. G. Delcey, L. Freitag, T. B. Pedersen, F. Aquilante, R. Lindh and L. González, J. Chem. Phys., 2014, 140, 174103.

45 A. Gavriluta, G. E. Büchel, L. Freitag, G. Novitchi, J. B. Tommasino, E. Jeanneau, P.-S. Kuhn, L. González, V. B. Arion and D. Luneau, Inorg. Chem., 2013, 52, 6260-6272.
46 K. Pierloot, Int. J. Quantum Chem., 2011, 111, 3291-3301.

47 J. P. Perdew, Phys. Rev. B: Condens. Matter Mater. Phys., 1986, 33, 8822-8824.

48 A. D. Becke, J. Chem. Phys., 1993, 98, 5648-5652.

49 F. Weigend and R. Ahlrichs, Phys. Chem. Chem. Phys., 2005, 7, 3297-3305.

50 K. Eichkorn, F. Weigend, O. Treutler and R. Ahlrichs, Theor. Chem. Acc., 1997, 97, 119-124.

51 D. Andrae, U. Häußermann, M. Dolg, H. Stoll and H. Preuß, Theor. Chim. Acta, 1990, 77, 123-141.

52 (a) K. Eichkorn, O. Treutler, H. Öhm, M. Häser and R. Ahlrichs, Chem. Phys. Lett., 1995, 240, 283-290; (b) M. Sierka, A. Hogekamp and R. Ahlrichs, J. Chem. Phys., 2003, 118, 9136-9148.

53 TURBOMOLE V6.5 2013, a development of University of Karlsruhe and Forschungszentrum Karlsruhe $\mathrm{GmbH}$, 1989-2007, TURBOMOLE GmbH, since 2007, available from http://www.turbomole.com.

54 B. O. Roos, R. Lindh, P.-A. Malmqvist, V. Veryazov and P.-O. Widmark, J. Phys. Chem. A, 2005, 109, 6575-6579.

55 F. Aquilante, L. de Vico, N. Ferré, G. Ghigo, P.-Å. Malmqvist, P. Neogrády, T. B. Pedersen, M. Pitoňák, M. Reiher, B. O. Roos, L. Serrano-Andrés, M. Urban, V. Veryazov and R. Lindh, J. Comput. Chem., 2010, 31, 224-247.

56 J. P. Perdew, K. Burke and M. Ernzerhof, Phys. Rev. Lett., 1996, 77, 3865-3868.

57 R. S. Mulliken, J. Chem. Phys., 1955, 23, 1833-1840.

58 S. F. Keller and M. Reiher, Chimia, 2014, 68, 200-203.

59 M. Dolfi, B. Bauer, S. Keller, A. Kosenkov, T. Ewart, A. Kantian, T. Giamarchi and M. Troyer, Comput. Phys. Commun., 2014, 185, 3430-3440.

60 F. Aquilante, T. B. Pedersen, A. Sánchez de Merás and H. Koch, J. Chem. Phys., 2006, 125, 174101.

61 Q. M. Phung, S. Vancoillie, A. Delabie, G. Pourtois and K. Pierloot, Theor. Chem. Acc., 2012, 131, 1-9. 